\title{
Smartphone / smartwatch-based cuffless blood pressure measurement : a position paper from the Korean Society of Hypertension
}

\author{
Hae Young Lee ${ }^{1 \dagger}$, Dong-Ju Lee ${ }^{1 \dagger}$, Jongmo Seo ${ }^{2}$, Sang-Hyun $\mathrm{hm}^{3}$, Kwang-il Kim ${ }^{4}$, Eun Joo Cho ${ }^{5}$, \\ Hyeon Chang Kim ${ }^{6}$, Jinho Shin', Sungha Park ${ }^{8}$, Il-Suk Sohn ${ }^{9}$, Wook-Jin Chung ${ }^{10}$, Sung Kee Ryu ${ }^{11}$, Ki Chul Sung ${ }^{12}$, \\ Juhan Kim ${ }^{13}$, Dae-Hee Kim ${ }^{14}$, Wook Bum Pyun ${ }^{15^{*}}$ and on behalf of the Korean Society of Hypertension
}

\begin{abstract}
Smartphone technology has spread rapidly around the globe. According to a report released by the Korea Information Society Development Institute, about 95\% of Koreans aged more than 30 years old owned smartphones. Recently, blood pressure (BP) measurement using a photoplethysmography-based smartphone algorithm paired with the smartwatch is continuously evolving. In this document, the Korean Society of Hypertension intends to remark the current results of smartphone / smartwatch-based BP measurement and recommend optimal BP measurement methods using a smartphone device. We aim to increase the likelihood of success in implementing these new technologies into improved hypertension awareness, diagnosis, and control.
\end{abstract}

Keywords: Blood pressure, Measurement, Hypertension, Smartphone, Plethysmography

\section{Background}

Higher blood pressure (BP) levels are associated with an increased risk of cardiovascular disease (CVD) and mortality [1]. Moreover, the probability of progression to hypertension (HTN) and the risk for a cardiovascular (CV) event was higher in the elevated BP or prehypertension groups than those in the normal BP group [2]. Although the overall management of HTN has improved over the past few decades, the rates of awareness, treatment, and control are recently stagnant even in the developed countries [3].

Accurate measurement of BP is essential for the diagnosis, treatment, and prognostication of individuals with

\footnotetext{
* Correspondence: pwb423@ewha.ac.kr

${ }^{\dagger}$ Hae Young Lee and Dong-Ju Lee contributed equally to this work.

${ }^{15}$ Division of Cardiology, Department of Internal Medicine, Ewha Womans University Seoul Hospital, Seoul, South Korea

Full list of author information is available at the end of the article
}

hypertension $[4,5]$. In the office or in the clinic, the auscultatory method of measuring BP using a stethoscope is still considered as a standard one. BP measuring devices include a mercury sphygmomanometer, an aneroid sphygmomanometer, and an electronic sphygmomanometer. The mercury sphygmomanometer has been replaced by an electronic or aneroid sphygmomanometer because of environmental mercury pollution. The importance of BP measurement outside the office has continuously empathized in clinical practice. Out-of-office BP measurement provides better prognostic information than office BP measurement alone [6]. Moreover, the self-measurement of BP can improve HTN awareness and also adherence [7].

Mobile technology has been widely adopted all over the world. Today, more than 5 billion people use mobile devices, and over a half of these are smartphones. According to a report by the Korea Information Society 
Development Institute in 2018 [8], 37.8\% of Koreans aged more than 70 years old owned smartphones in 2018 , comparing $3.6 \%$ in 2013 . The number of smartphone users increased over the last 5 years, with the number for people in their 60 s rose from 19 to $80.3 \%$, 50s from 51.3 to $95.5 \%, 40$ s 81.3 to $98.4 \%$, and 30 s 94.2 to $98.7 \%$. Indeed, the percentage of adults who own smartphones is higher in South Korea than in any other developed countries (95\%), including Israel (88\%) and the Netherlands (87\%) [9]. Even before the emergence of smartphones, various wearable devices were developed to estimate BP by photoplethysmographic sensors and signal analysis [10]. However, shortly after the introduction of the conventional smartphone in 2007 (iPhone) and 2008 (Android), it was soon discovered that the smartphone camera could be used as a photoplethysmographic sensor to obtain a signal waveform [10]. Recently, BP measurement using 'cuffless' photoplethysmography-based smartphone algorithm paired with the smartwatch is continuously evolving. Indeed, recent smartwatch devices fulfilled the requirement for a medical device in the International Organization for Standardization (ISO) 81,060-2:2018 Non-invasive sphygmomanometers [11].

In this document, the Korean Society of Hypertension intends to remark the current results of smartphone / smartwatch-based BP measurement and recommend optimal BP measurement using a smartphone device. We aim to describe the current limitations and requirements to guide the successful implementation of these new technologies for improving HTN awareness, diagnosis, and control.

\section{Part 1. Current results of smartphone-based blood pressure measurement \\ Data source and searches}

The Pubmed database was systemically searched for studies published from January 2010 through July 2020. Our Pubmed search query was 'smartphone' or 'smart phone' and 'blood pressure', explicitly focusing on title/ abstract. We included every paper until July 2020. Not only English but also every paper in other languages were included. Also, all references from selected papers were therefore assessed for relevant studies.

Studies retrieved from the initial database search were examined by predetermined selection criteria. Inclusion criteria were as follows: (1) related to direct BP measurement, and the measurement method is different from the conventional method; (2) smartphone is used as a BP measuring device; (3) original paper that conducted experiments on real people. The exclusion criteria were as follows: (1) an application (App) encouraging BP measurement; (2) principle of oscillometric or sphygmomanometer are used as a measurement method; (3) Self- manufactured equipment; (4) Review papers. Observational studies, study protocols and designs, studies with an abstract presentation, and duplicates were all excluded.

\section{Search results}

A total of 368 papers were initially identified from the Pubmed database; 34 duplicated papers were excluded. The remaining 334 studies were then screened by two independent cardiologists. We excluded 293 papers because of the low relevance of the title and abstract of this paper. A further 11 papers were excluded because they did not evaluate the smartphone as a BP measurement device. After reviewing the full text of the remaining 30 papers, 18 papers were excluded due to reviews or nonhuman studies. All of the 12 papers were included in a narrative synthesis. A flow diagram of the selected studies is shown in Fig. 1.

\section{Study characteristics}

The characteristics of the 12 papers are shown in Table 1. Six studies were conducted in general subjects with normal BP, and the other six studies evaluated in subjects with varying BP ranged. Seven studies used iPhones as a BP-measuring device, three for Galaxy, and the rest for Google Pixel, Android, and Nexus, respectively. Four studies used a sphygmomanometer as a comparison method, while eight used the oscillometric method, and one used a finger sensor.

Two studies satisfied the Association for the Advancement of Medical Instrumentation (AAMI) criteria [24], which indicates that only these studies can be used as a valid $\mathrm{BP}$ measuring instrument. The other ten studies did not meet the criteria. In a paper on various BP studies, the more the $\mathrm{BP}$ values deviated from normal, the larger the error was. In addition, all studies have stated that further studies are warranted on the limitation of the insufficient number of samples and how accurate results will be obtained when this is done in real human subjects.

In the case of the early developmental period implementing smartphones as BP measuring devices by using the heartbeat and photoplethysmographic wave analysis combined with the computerized algorithm, the accuracy was between $95 \sim 100 \%$. However, there were large fluctuations depending on the method of measurement [12]. Therefore, the measurement results were presented as $\mathrm{BP}$ ranges rather than actual $\mathrm{BP}$ values. In that case of describing the accuracy by $\mathrm{BP}$ range, F-scores of 0.78 and 0.8 \{systolic BP (SBP) and diastolic BP (DBP)\} were higher than using actual values [13].

Afterward, many studies have been conducted to use smartphones as BP measuring devices. However, there were no satisfactory results proving consistency 


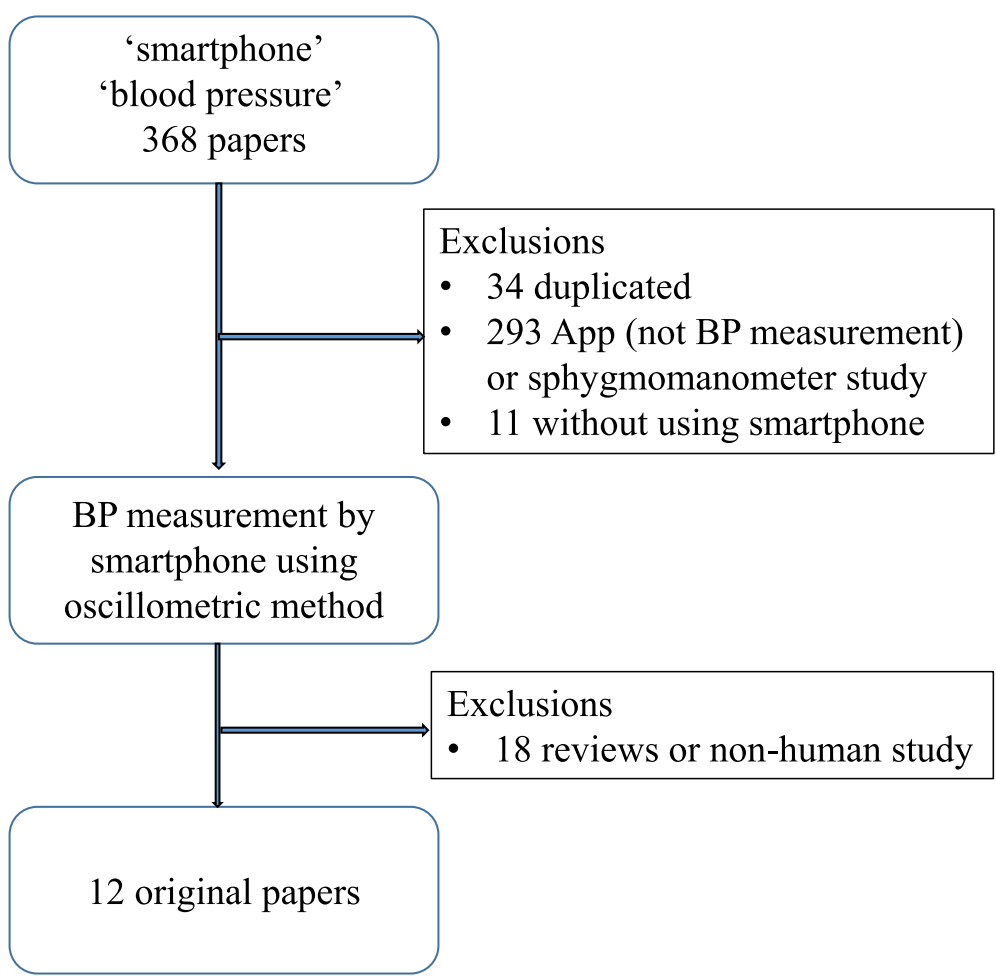

Fig. 1 Study selection

Table 1 Summary of smartphone / smartwatch-based cuffless blood pressure measurement

\begin{tabular}{|c|c|c|c|c|c|c|c|}
\hline \multirow[t]{2}{*}{ Year } & \multirow[t]{2}{*}{ Authors } & \multirow[t]{2}{*}{ Subjects } & \multirow[t]{2}{*}{ Type of subjects } & \multirow[t]{2}{*}{ Device } & \multirow{2}{*}{$\begin{array}{l}\text { Comparison (Measuring } \\
\text { location / device) }\end{array}$} & \multicolumn{2}{|c|}{ Mean $\pm \mathrm{SD}(\mathrm{mmHg})$} \\
\hline & & & & & & SBP & DBP \\
\hline 2013 & Chandrasekaran, et al. [12] & 500 & Healthy subjects & Nexus One & $\begin{array}{l}\text { Brachial cuff / Mercury } \\
\text { sphygmomanometer }\end{array}$ & $\pm 2.4473(95 \% \mathrm{Cl})$ & $\pm 1.7073(95 \% \mathrm{Cl})$ \\
\hline 2014 & Visvanathan, et al. [13] & 156 & Variable subjects & Iphone4 & $\begin{array}{l}\text { Brachial cuff / ETCOMM } \\
\text { HC-502 }\end{array}$ & 0.78 (F-score) & 0.80 (F-score) \\
\hline 2016 & Plante, et al. [14] & 85 & Variable subjects & iPhone $5 \mathrm{~s}, 6$ & $\begin{array}{l}\text { Brachial cuff / Omron 907, } \\
907 X L\end{array}$ & $12.4 \pm 10.5$ & $10.1 \pm 8.1$ \\
\hline 2016 & Gaurav, et al. [15] & 3000 & Healthy subjects & galaxy note 5 & $\begin{array}{l}\text { Brachial cuff / Mercury } \\
\text { sphygmomanometer }\end{array}$ & $0.16 \pm 6.85$ & $0.03 \pm 4.72$ \\
\hline 2016 & Alexander, et al. [16] & 100 & Healthy subjects & iPhone $5 \mathrm{~s}$ & $\begin{array}{l}\text { Brachial cuff / Welch Allyn } \\
\text { Propaq CS Monitor }\end{array}$ & $-0.6 \pm 12.8$ & $+7.2 \pm 9.2$ \\
\hline 2016 & Gao, et al. [17] & 78 & Healthy subjects & Android phone & $\begin{array}{l}\text { Brachial cuff / A\&D UA- } \\
\text { 767PBT }\end{array}$ & $5.1 \pm 4.3$ & $4.6 \pm 4.3$ \\
\hline 2018 & Raichle, et al. [18] & 32 & Variable subjects & Iphone $4 \mathrm{~s}$ & $\begin{array}{l}\text { Brachial cuff / Omron- } \\
\text { HBP-1300 }\end{array}$ & $5.0 \pm 14.5$ & NA. \\
\hline 2018 & Wang, et al. [19] & 7 & Variable subjects & Google pixel phone & $\begin{array}{l}\text { Brachial cuff / Microlife } \\
\text { BP3NA1-1x }\end{array}$ & N.A. & $4.8 \pm 4.7$ \\
\hline 2018 & Chandrasekaran, et al. [20] & 20 & Healthy subjects & iPhone X & $\begin{array}{l}\text { Brachial cuff / Omron } \\
\text { BP786 }\end{array}$ & $-4.0 \pm 11.4$ & $-9.4 \pm 9.7$ \\
\hline 2018 & Dey, et al. [21] & 205 & Variable subjects & Galaxy S6 & $\begin{array}{l}\text { Brachial cuff / Mercury } \\
\text { sphygmomanometer }\end{array}$ & $6.90 \pm 9.00$ & $5.00 \pm 6.10$ \\
\hline 2018 & Matsumura, et al. [22] & 49 & Variable subjects & iPhone $6 \mathrm{~s}$ & $\begin{array}{l}\text { Brachial cuff / NISSEI, DS- } \\
\text { S10 }\end{array}$ & $0.67 \pm 12.7$ & $0.45 \pm 8.6$ \\
\hline 2019 & Luo, et al. [23] & 1328 & Healthy subjects & iPhone 6+ & Finger sensor / CNAP 500 & $0.39 \pm 7.3$ & $-0.20 \pm 6.00$ \\
\hline
\end{tabular}


with the standard BP measured by standard brachial BP measured by sphygmomanometers satisfying the AAMI standard [14, 15, 17-19].

However, recent studies have shown much-improving data by applying ubiquitous models or linear polynomial equation [21, 22]. Finally, the proof-of-concept study applying an iPhone camera sensor for BP monitoring via the oscillometric finger pressing method showed bias and precision errors of -4.0 and $11.4 \mathrm{mmHg}$ for SBP and -9.4 and $9.7 \mathrm{mmHg}$ for DBP [20]. These errors were near the finger cuff device errors, which is FDA-cleared for measuring brachial BP $[25,26]$. Furthermore, this smartphone-based BP measurement using transdermal optical imaging technology method results in normotensive adults fall within $5 \pm 8 \mathrm{mmHg}$ of reference measurements, which satisfied a key accuracy threshold bias and SD when testing proceeds according to the AAMI standard $[23,24]$.

There are two critical concerns about BP measurement using a smartwatch device. One is that the accuracy of the photoplethysmographic sensor-based BP measurement is not validated. The existence of surrounding near-infrared (NIR) light sources could disturb accurate measurement [10]. Another problem is that there is low reliability of wrist BP because of an error in home self-measurement at the wrist despite appropriate training [27]. Although the data were from the cuffinflation method of wrist BP, an appropriate static position is difficult in wrist measurement.

In summary, recent studies of BP measurement using a smartwatch device reported acceptable accuracy within $5 \pm 8 \mathrm{mmHg}$ of reference measurements, which satisfied a key accuracy threshold bias and SD when testing proceeds according to the AAMI standard. However, there is concern of wrist BP self-measurement at home even despite appropriate training.

\section{Part 2. Recommendations of blood pressure measuring method using smartwatch device}

In order to use smartwatches as BP measuring device, it is mandatory to calibrate $\mathrm{BP}$ inputting the user's $\mathrm{BP}$ values obtained by the standard BP monitoring device regularly. This calibration process might have the ancillary benefit to increase the awareness of BP. During the calibration process, it is recommended to measure BP by three times at least $2 \mathrm{~min}$ apart. Also, when the user changes the wrist wearing a smartwatch, the calibration process should be repeated.

The major issue in the calibration process is the difference in $\mathrm{BP}$ between the two arms. The mean inter-arm difference was $3.3 \mathrm{mmHg}$ for SBP and $2.0 \mathrm{mmHg}$ for DBP in extensive epidemiologic studies [28]. An increased inter-arm SBP difference $(\geq 10 \mathrm{mmHg})$ is found in $5-10 \%$ of the population, associated with a future increase in SBP or progression to HTN [29]. Therefore, if the calibration was performed based on the BP values measured in the opposite arm, this can cause at least 3 $\mathrm{mmHg}$ error, which cannot be corrected by any internal calibration mechanism.

In the clinical trial, the research coordinators could help the subjects calibrate in the opposite arm while measuring BP using a smartwatch device. In this setting, simultaneous calibration using the opposite arm can minimize the temporal variation of BP. However, in the real world situation, the user cannot calibrate simultaneously without assistance, and then there is no benefit using the opposite arm for the calibration. Therefore, the experts recommend calibrating the smartwatch device using the same arm measurement of $\mathrm{BP}$ after acquiring photoplethysmography-based pulse signals in the smartwatch sequentially. It is crucial to acquire acquiring photoplethysmography-based pulse signals first because upper arm compression by cuff can influence the pulse signals by hyperemia [30]. In the American Heart Association BP measurement guideline [31], separate BP measurement repeated by $1-2 \mathrm{~min}$ is recommended. Venous congestion or hyperemia has traditionally been thought to affect the BP measurement results, especially when the Korotkoff method is used. However, in recent reports, very short time intervals between readings did not produce different values from conventional intervals when oscillometric devices were used [32].

The recommendation of the traditional cuff-based BP measurement method should be commonly applied to the smartwatch device, especially if the BP value was used for the diagnosis and the treatment of $\mathrm{HTN}^{4}$. The overview of proper seated BP measurement using a smartwatch device was summarized in Table 2.

In addition, the accuracy may be further reduced in patients with several medical conditions: aortic valve insufficiency with wide pulse pressure, atrial fibrillation with considerable beat-to-beat variability, peripheral vascular disease with weak perfusion, diabetes, cardiomyopathy, ESRD, neurological disorders such as hand tremor, blood clotting disorder, or taking antiplatelet agents / anticoagulants. It is also not recommended for pregnant women as the vascular characteristics differ from those of the general population due to the large hormonal changes (sudden and dramatic increases in estrogen \& progesterone) during pregnancy [18].

In summary, the adequate training of BP selfmeasurement by standard method summarized in Table 2 is essential part for the accuracy. BP measurement using a smartwatch device is not recommended in patients with several medical conditions, high $\mathrm{SBP} \geq 160$ $\mathrm{mmHg}$, or low $\mathrm{BP} \leq 60 \mathrm{mmHg}$. 


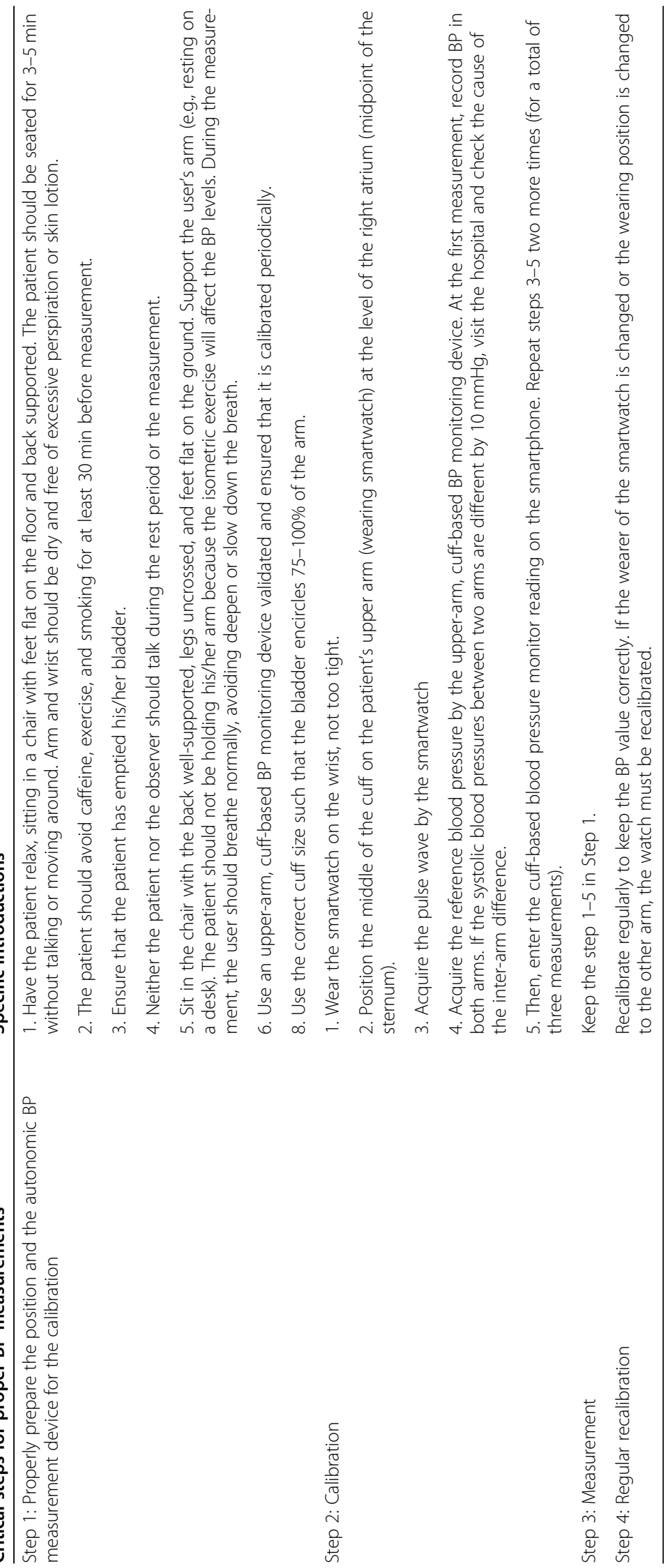




\section{Part 3. Gaps in the evidence and need for further studies}

Currently, the purpose of $\mathrm{BP}$ measurement using a smartwatch device is likely to increase awareness of $\mathrm{BP}$ and possibly to detect HTN early in the general population, rather than to monitor treatment response in hypertensive patients. Because the current standard of AAMI validated device includes a calibration process, for the time being, accessibility to those devices seems to be low in the older generations. In contrast, considering that low awareness rate of HTN in those in the 30s to 40s age groups who may be more accustomed to those devices, smartwatch-based BP measurement can offer an opportunity to make young adults pay attention to high BP and start HTN treatment early.

However, it is well expected that hypertensive patients will use smartwatch devices for BP monitoring either in resting conditions or stress conditions. There is still scanty evidence in the use of a smartwatch device for hypertensive patients. Indeed, recent smartwatch devices fulfilled the requirement for a medical device in ISO 81060-2:2018 Non-invasive sphygmomanometers, socalled AAMI standard [11]. Although the clinical study enrolled the subjects whose gender, age, and ethnicity distribution were well satisfied with ISO criteria, only $30 \%$ of the subjects were hypertensive, and the measurement error increase according to BP increase. Therefore, the feasibility of the smartwatch device use in HTN management should be tested in many steps of clinical studies.

In terms of "usual BP" controversy, it could be reconsidered if a new standard for the validation of the smartwatch device to measure BP in an ambulatory or daily living setting is a need. In other words, when a device calibrated at resting condition, which is supposed to be used in an ambulatory setting, the accuracy of BP measured in an ambulatory setting may not be extrapolated from the calibration data. Because the reference value for the validation of a $\mathrm{BP}$ measuring device is only for the resting status regardless of invasive or non-invasive methods, how ambulation or non-resting condition would have impacts on the calibration parameters for smartwatch needs to be incorporated to the resting calibration data if it is possible to quantify the degree of ambulation or non-resting states. Alternatively, we might use the reference value established during the ambulatory setting, even though it does not seem feasible. In that case, it could be applied to the validation of a smartwatch to measure BP during daily living directly with the big data-based calibration to overcome the increased number of calibration parameters in various ambulatory situations. Therefore, the consensus on the need for new validation reference for smartwatch devices and its development will have a big impact on $\mathrm{BP}$ measurement and its related research fields.

Likely, the use of self-monitoring for the routine evaluation of hypertensive patients will be accelerated using the smartwatch devices. This trend may be recommended as part of a general movement in which patients play an increasingly important role in the management of their health. Poor adherence to therapy has been recognized as one of the most critical factors contributing to uncontrolled HTN. Knowing patients' BP for themselves will be the most important way of improving patient's adherence to lifestyle changes and/or medical treatment. In contrast, if patients titrated their medication based on the inadequate BP measurement, there might be harms such as doubling or skipping a dose. Importantly, self-monitoring of BPs were not evenly distributed in $24 \mathrm{~h}$ or 7 days, or even seasons [33]. Especially there are concerns that high BP values measured by inaccurate methods might result in unnecessary psychological stress, false-labeling of HTN, and further overspending on medical expenses [34]. The costeffectiveness analysis of BP measurement using a smartwatch is necessary at some point.

However, BP measurements using a smartwatch might open a new field of dynamic evaluation of BP. Although absolute $\mathrm{BP}$ values appear to be the most important factors determining prognosis, BP variability (BPV) has also been proven in many studies to be an independent and robust indicator of a future cardiovascular events [35]. However, conventional BP measurement, even including ambulatory blood pressure measurement, mainly focused on the resting state's static BP measurement. However, the smartphone / smartwatch-based BP measurement will naturally report BP change in daily living and physical/emotional stress, thus opening a new chapter of $\mathrm{BP}$ research regarding dynamic $\mathrm{BP}$ change. BPV is higher during weekdays and winter season, supporting that environmental factors such as job stress and the outside environment influence BPV [33]. With a more comprehensive application of smartphone / smartwatchbased cuffless BP measurement, the research about BPV in dynamic conditions will be brisk.

In summary, current status of BP measurement using a smartwatch device is for increasing awareness of BP and possibly detecting HTN early in the general population. And it is still not recommended to use BP measurement using a smartwatch device for monitoring treatment response in hypertensive patients.

\section{Conclusion}

Smartphone technology has spread rapidly around the globe. Moreover, BP measurement using a photoplethysmography-based smartphone algorithm paired with a smartwatch will continue to grow in the 
foreseeable future. No one can be irresistible against this flow of the times. Recently, BP measurement using a photoplethysmography-based smartphone algorithm paired with a smartwatch is approved as a medical device, fulfilled ISO standards. The current results showed acceptable accuracy in the study population. However, there is still a considerable discrepancy in high or low $\mathrm{BP}$ ranges than the conventional $\mathrm{BP}$ measurement. The use of smartwatch-based BP measurement can improve HTN awareness, and especially in the younger population. However, there is still scanty evidence in the use of smartwatch devices for hypertensive patients. The convenience and easy-to-use is the motto of the smartphone / smartwatch device; however, it might be a 'doubleedged sword' if the measurement were not correctly performed. Therefore, adequate education of the BP measurement method is essential to maximize benefit. Future efforts (and collaborations) should also be made by both researchers and companies to evaluate the effectiveness and usability of medical devices for the hypertensive population.

\section{Acknowledgements}

We thank Jongmin Choi and Sunok Jung (Samsung Electronics) for technical assistance in Galaxy watch device instruction.

\section{Authors' contributions}

HYL and DJL wrote the draft. JMS, SHI, KK, EJC, HCK, JS, SP, ISS, WJC, SKR, KCS, JK, and DHK reviewed and edited the manuscript. WBP administrated this position paper. All authors have read and agreed to the published version of the manuscript.

\section{Funding}

None.

\section{Availability of data and materials}

Published medical data mostly of last 5 years.

\section{Ethics approval and consent to participate}

Not applicable.

\section{Consent for publication}

Not applicable.

\section{Competing interests}

Nil.

\section{Author details}

'Seoul National University College of Medicine, Seoul, South Korea. ${ }^{2}$ Department of Electrical and Computer Engineering, College of Engineering, Seoul National University, Seoul, South Korea. ${ }^{3}$ Division of Cardiology, Department of Internal Medicine, Bucheon St. Mary's Hospital, College of Medicine, The Catholic University of Korea, Seoul, South Korea. ${ }^{4}$ Division of Geriatrics, Department of Internal Medicine, Seoul National University Bundang Hospital, Seongnam, South Korea. ${ }^{5}$ Division of Cardiology, Department of Internal Medicine, Yeouido St. Mary's Hospital, College of Medicine, The Catholic University of Korea, Seoul, South Korea. ${ }^{6}$ Department of Preventive Medicine, Yonsei University College of Medicine, Seoul, South Korea. ${ }^{7}$ Division of Cardiology, Department of Internal Medicine, Hanyang University Medical Center, Seoul, South Korea. ${ }^{8}$ Division of Cardiology, Severance Cardiovascular Hospital and Severance Cardiovascular Hospital and Integrated Research Center for Cerebrovascular and Cardiovascular Diseases, Yonsei University College of Medicine, Seoul, South Korea. ${ }^{9}$ Division of Cardiology, Department of Internal Medicine, Kyung Hee University at Gangdong, Seoul, South Korea. ${ }^{10}$ Division of Cardiology,
Department of Internal Medicine, Gachon University Gil Medical Center, Incheon, South Korea. ${ }^{11}$ Division of Cardiology, Department of Internal Medicine, Nowon Eulji Medical Center, Eulji University, Seoul, South Korea. ${ }^{12}$ Division of Cardiology, Department of Internal Medicine, Kangbuk Samsung Hospital, Seoul, South Korea. ${ }^{13}$ Division of Cardiology, Department of Internal Medicine, Chonnam National University Hospital, Gwangju, South Korea.

${ }^{14}$ Department of Cardiology, Asan Medical Center, College of Medicine, Ulsan University, Seoul, South Korea. ${ }^{15}$ Division of Cardiology, Department of Internal Medicine, Ewha Womans University Seoul Hospital, Seoul, South Korea

Received: 25 September 2020 Accepted: 23 November 2020

Published online: 25 January 2021

\section{References}

1. Kim HC, Ihm SH, Kim GH, Kim JH, Kim KI, Lee HY, et al. 2018 Korean Society of Hypertension guidelines for the management of hypertension: part 1epidemiology of hypertension. Clin Hypertens. 2019;25:16.

2. Kim SJ, Lee J, Nam CM, Jee SH, Park IS, Lee KJ, et al. Progression rate from new-onset pre-hypertension to hypertension in Korean adults. Circ J. 2011; 75(1):135-40.

3. Collaboration NCDRF. Long-term and recent trends in hypertension awareness, treatment, and control in 12 high-income countries: an analysis of 123 nationally representative surveys. Lancet. 2019:394(10199):639-51.

4. Kim Kl, Ihm SH, Kim GH, Kim HC, Kim JH, Lee HY, et al. 2018 Korean society of hypertension quidelines for the management of hypertension: part IIIhypertension in special situations. Clin Hypertens. 2019;25:19.

5. Lee HY, Shin J, Kim GH, Park S, Ihm SH, Kim HC, et al. 2018 Korean Society of Hypertension Guidelines for the management of hypertension: part IIdiagnosis and treatment of hypertension. Clin Hypertens. 2019;25:20.

6. Verberk WJ, Kroon AA, Lenders JW, Kessels AG, van Montfrans GA, Smit AJ et al. Self-measurement of blood pressure at home reduces the need for antihypertensive drugs: a randomized, controlled trial. Hypertension. 2007; 50(6):1019-25.

7. Cappuccio FP, Kerry SM, Forbes L, Donald A. Blood pressure control by home monitoring: meta-analysis of randomised trials. BMJ. 2004;329(7458): 145.

8. Institute KISD. 2018 Korea media panel survey; 2018.

9. Taylor KSL. Smartphone ownership is growing rapidly around the world, but not always equally. Pew research center; 2019.

10. Elgendi $M$, Fletcher R, Liang $Y$, Howard N, Lovell NH, Abbott D, et al. The use of photoplethysmography for assessing hypertension. NPJ Digit Med. 2019;2:60

11. Standardization IOf. ISO 81060-2:2018(en). Non-invasive sphygmomanometers - Part 2: Clinical investigation of intermittent automated measurement type. https://www.iso.org/obp/ui/\#iso:std:iso:8106 0:-2:ed-3:v1:en.

12. Chandrasekaran V, Dantu R, Jonnada S, Thiyagaraja S, Subbu KP. Cuffless differential blood pressure estimation using smart phones. IEEE Trans Biomed Eng. 2013;60(4):1080-9.

13. Visvanathan ABR, Dutta Choudhury A, Sinha A, Kundu S. Smart phone based blood pressure indicator. In: Proceedings of the 4th ACM MobiHoc workshop on pervasive wireless healthcare; 2014. p. 19-24.

14. Plante TB, Urrea B, MacFarlane ZT, Blumenthal RS, Miller ER 3rd, Appel $L$, et al. Validation of the instant blood pressure smartphone app. JAMA Intern Med. 2016;176(5):700-2.

15. Gaurav AMM, Tiwari VN, Narayanan R. Cuff-less PPG based continuous blood pressure monitoring - a smartphone based approach. In: 2016 38th annual international conference of the IEEE engineering in medicine and biology society (EMBC); 2016. p. 607-10.

16. Alexander JC, Minhajuddin A, Joshi GP. Comparison of smartphone application-based vital sign monitors without external hardware versus those used in clinical practice: a prospective trial. J Clin Monit Comput. 2017;31(4):825-31

17. Gao SCWP, Zhao L, Jiang WJ. Data-driven estimation of blood pressure using photoplethysmographic signals. In: 2016 38th annual international conference of the IEEE engineering in medicine and biology society (EMBC); 2016. p. 766-9.

18. Raichle CJ, Eckstein J, Lapaire O, Leonardi L, Brasier N, Vischer AS, et al. Performance of a blood pressure smartphone app in pregnant women: the 
iPARR trial (iPhone app compared with standard RR measurement). Hypertension. 2018;71(6):1164-9.

19. Wang EJZJ, Jain M, Lee T-J, Saba E, Nachman L, Patel SN. Seismo: blood pressure monitoring using built-in smartphone accelerometer and camera. In: Proceedings of the $2018 \mathrm{CHI}$ conference on human factors in computing Systems; 2018. p. 1-9.

20. Chandrasekhar A, Natarajan K, Yavarimanesh M, Mukkamala R. An iPhone application for blood pressure monitoring via the Oscillometric finger pressing method. Sci Rep. 2018;8(1):13136.

21. Dey JGA, Tiwari VN. Instabp: cuff-less blood pressure monitoring on smartphone using single ppg sensor. In: 2018 40th annual international conference of the IEEE engineering in medicine and biology society (EMBC); 2018. p. 5002-5.

22. Matsumura K, Rolfe P, Toda S, Yamakoshi T. Cuffless blood pressure estimation using only a smartphone. Sci Rep. 2018;8(1):7298.

23. Luo H, Yang D, Barszczyk A, Vempala N, Wei J, Wu SJ, et al. Smartphonebased blood pressure measurement using transdermal optical imaging technology. Circ Cardiovasc Imaging. 2019;12(8):e008857.

24. Instrumentation. AftAoM. ANSI/AAMI/ISO 81060-1:2007/(R)2013.

25. Systems FFM. The Finapres NOVA has received $510(\mathrm{k})$ clearance from the US FDA! 2018.

26. Heeney ND, Habib F, Brar GK, Krahn G, Campbell DA, Sanatani S, et al. Validation of finger blood pressure monitoring in children. Blood Press Monit. 2019;24(3):137-45.

27. Casiglia E, Tikhonoff V, Albertini F, Palatini P. Poor reliability of wrist blood pressure self-measurement at home: a population-based study. Hypertension. 2016;68(4):896-903.

28. Song BM, Kim HC, Shim JS, Lee MH, Choi DP. Inter-arm difference in brachial blood pressure in the general population of Koreans. Korean Circ J. 2016;46(3):374-83.

29. Weinberg I, Gona P, O'Donnell CJ, Jaff MR, Murabito JM. The systolic blood pressure difference between arms and cardiovascular disease in the Framingham heart study. Am J Med. 2014;127(3):209-15.

30. Rabbany SY, Drzewiecki GM, Noordergraaf A. Peripheral vascular effects on auscultatory blood pressure measurement. J Clin Monit. 1993;9(1):9-17.

31. Muntner P, Shimbo D, Carey RM, Charleston JB, Gaillard T, Misra S, et al. Measurement of blood pressure in humans: a scientific statement from the American Heart Association. Hypertension. 2019;73(5):e35-66.

32. Koehler NR. Poli de Figueiredo CE, Mendes-Ribeiro AC. Time interval between pairs of arterial blood pressure measurements--does it matter? Am J Hypertens. 2004;17(2):194-6.

33. Kim Kl, Nikzad N, Quer G, Wineinger NE, Vegreville M, Normand A, et al. Real world home blood pressure variability in over 56,000 individuals with nearly 17 million measurements. Am J Hypertens. 2018;31(5):566-73.

34. de Tuero GC, Boreu QF, Rodriguez-Poncelas A, Creus R, Sanmartin M, Salleras $\mathrm{N}$, et al. Assessment of self-monitoring of blood pressure in the diagnosis of isolated clinic hypertension. Blood Press. 2006;15(4):227-36.

35. Diaz KM, Tanner RM, Falzon L, Levitan EB, Reynolds K, Shimbo D, et al. Visitto-visit variability of blood pressure and cardiovascular disease and all-cause mortality: a systematic review and meta-analysis. Hypertension. 2014;64(5): $965-82$

\section{Publisher's Note}

Springer Nature remains neutral with regard to jurisdictional claims in published maps and institutional affiliations.

Ready to submit your research? Choose BMC and benefit from:

- fast, convenient online submission

- thorough peer review by experienced researchers in your field

- rapid publication on acceptance

- support for research data, including large and complex data types

- gold Open Access which fosters wider collaboration and increased citations

- maximum visibility for your research: over $100 \mathrm{M}$ website views per year

At BMC, research is always in progress.

Learn more biomedcentral.com/submissions 